CLINICAL ETHICS

\title{
Doctors' views about the importance of shared values in HIV positive patient care: a qualitative study
}

\author{
A Lawlor, A Braunack-Mayer
}

J Med Ethics 2004;30:539-543. doi: 10.1136/jme.2002.001560

Robert Veatch has proposed a model of the doctor-patient relationship that has as its foundation the sharing of values between the doctor and the patient. This paper uses qualitative research conducted with six doctors involved in the long term, specialised care of HIV positive patients in South Australia to explore the practical application of Veatch's value sharing model in that setting. The research found that the doctors in this study linked "values" with sexual identity such that they defined value sharing, in part, as a shared set of values and beliefs about sexual identity and practices. They voluntarily identified themselves as either homosexual or heterosexual and they regarded the relation between their own sexual identity and that of their patients as important for the provision of quality care. None of the doctors thought that value sharing, in the way they defined it, was essential to the clinical relationship, but the homosexual doctors attributed a greater degree of importance to it than their heterosexual colleagues.

See end of article for authors' affiliations

Correspondence to: $\operatorname{Dr}$ A Braunack-Mayer, Lecturer in Ethics, Department of Public Health, University of Adelaide, SA 5005, Australia; annette. braunackmayer@ adelaide.edu.au

Received 27 August 2002 In revised form 22 April 2003

Accepted for publication

29 June 2003
$\mathrm{T}$ he debate surrounding the concept of "value" has a long and complex history. As Hart points out "... valuational issues have been with us the moment man began to reflect upon conditions of his life, the structure of reality, the order of nature and man's place in it." ${ }^{1}$ Value classification systems vary in content and complexity. ${ }^{2-4}$ The difficulty inherent in any discussion of values, as Misztal puts it is "the number and variety of hierarchical value systems adopted by societies and individuals". ${ }^{5}$

The idea of "value sharing" is likewise not a new one. Although definitions of "value" vary, there is a clear sense among authors writing about this topic that the perception of shared values is important to group membership processes $^{6}$ and the development of social and group identities. $^{7}$ The perception of similar values in others is what draws a person to a group and forms the basis of group interactions. ${ }^{8}$ As a mediating factor in interpersonal relations, value sharing is potentially important in the medical setting, as the delivery of medical care is often achieved by the patient deciding to embark upon and/or maintain a relationship with a particular provider.

Robert Veatch has proposed a model of the clinical relationship which has as its foundation the sharing of values between the doctor and the patient. Veatch claims that:

"It seems unfair to patients to have choices about their health and well-being made based on someone else's values, especially when we know that other clinicians would have drawn on other values."

The values to which Veatch refers are not only medical values, though he certainly recognises the importance of medical values in medical decision making. It is the non-medical values that Veatch sees as being particularly important, those originating in "sources well beyond medicine". ${ }^{10}$ These values cover a number of dimensions of a person's life. For example, they include “... religion, social philosophy, socioeconomic and political persuasion, or moral instinct."11 Veatch's strategy for overcoming this ethical difficulty of making choices in what the doctor believes to be the patient's best interest, is what he terms "value matching"; that is, "patients and clinicians pair up purposely based on values". ${ }^{12}$

Doctors all have value systems which inform the choices they make and the people they are. ${ }^{13}$ The premise for Veatch's strategy is that patients are experts in the determination of what is in their best interests based on their own personal value systems. Doctors-irrespective of intentare poorly qualified to make these types of decisions. In pairing a patient with a doctor who shares that patient's values, Veatch suggests that decisions made by the doctor based on his/ her assessment of the patient's interests will closely approximate the choices the patient would have made. ${ }^{14}$

This paper explores the application of the value sharing model in a single setting: the care of HIV positive patients in South Australia. This setting provided a unique opportunity to study the doctor-patient relationship for two reasons. Value judgements, along with fear and ignorance, have played an important part in the stigmatisation and marginalisation HIV positive people report having experienced. Despite a gradually declining prejudice over the last two decades, HIV positive patients remain a stigmatised and marginalised group. ${ }^{15}{ }^{16}$ Negative attitudes to people with HIV/AIDS are also to be found in alarmingly high proportions among medical and allied health professionals. ${ }^{17}{ }^{18}$

Abbreviation: GHAM, gay and other homosexually active men 
Because the social difficulties HIV positive patients face are largely based on negative value judgements, doctors' considerations of issues surrounding values in HIV are particularly relevant.

Secondly, there were also local contextual reasons for the focus on the care of HIV positive patients. The specialised long term care of HIV positive patients in South Australia is carried out by a small number of doctors. As such, by interviewing the majority of these clinicians, the attitudes of those doctors who care for a large proportion of HIV positive patients in South Australia could be assessed.

The aim of this study, then, was to determine the extent to which doctors working with HIV positive patients believe sharing patients' values is important in providing optimal health care.

\section{METHODS}

A list of doctors providing care to HIV positive patients in South Australia was compiled. Six doctors were identified as providing the majority of the long term, specialised HIV care in this state. These doctors were contacted by telephone, initially by a general practitioner working in HIV care and then by the interviewer (AL), and invited to participate in the study. The purpose of the study was explained to each prospective participant and an appointment time set at which the doctors could be interviewed. All six doctors agreed to be interviewed.

Of the six doctors, four were general practitioners and two were sexual health physicians. Each of the doctors had been involved in the care of HIV positive patients for 11 years or longer. The collective HIV positive patient load of the six doctors was estimated to account for approximately $75 \%$ of the known HIV positive patients in South Australia with the remaining $25 \%$ thought to be irregular users of health services.

Semi-structured interviews, lasting from one to about one and a half hours, were undertaken individually with the six doctors. The doctors were asked to discuss a range of practical and attitudinal features of their care for HIV positive patients. Box 1 summarises the major categories of questions. Four of the interviews were recorded on audiocassette and transcribed. Environmental noise made audio recording of the remaining two interviews impossible. Records of these interviews were therefore made by means of detailed note taking. The resulting transcripts varied in length from six to 22 pages.

The analysis of the interview records was undertaken using a process of analytic induction. NUD*IST, a qualitative data analysis package, was used to code response categories and classify information on common theme areas. ${ }^{19}$ Individual responses were then compared for the degree of concord or variance amongst respondents.

\section{RESULTS}

Each doctor was asked about the major risk factors responsible for HIV infection in their respective HIV positive patient populations. In all but one case, the vast majority of HIV positive patients were gay and other homosexually active men (GHAM). This included gay-identified and bisexual men and men who did not subscribe to either label but who reported a history of sexual contact with other men. The remaining respondent had extensive past experience working with HIV positive GHAM but reported a range of risk factors for his current patients including male to male sexual contact, intravenous drug use, and heterosexual sex overseas.

One important aspect of the analysis presented below requires explanation at this point. The results are presented, in part, as a comparison between the views of doctors who were identified as homosexual and those who were
Box 1 Semi-structured interview question categories

HIV care provision

- Patient population worked with

- Nature of work

- Health care needs of your HIV positive patients

- Compare with health care needs of non-HIV positive patients

- Qualities HIV positive patients are looking for in their doctors

- Skills/qualities for doctors to provide optimal health care to HIV positive patients

- How patients get access to good care, and ways to do this

Barriers to quality care

- Limits to appropriate care for HIV positive patients

- Barriers which restrict doctors' ability to provide quality health care

Shared values

- HIV positive patients' values and value systems

- Characteristics of patients' values and evolution of values

- Extent to which doctor shares values with patients

- Should doctors share values? Why? Why not?

- Impact on patient care

- Barriers to patients disclosing values

- Teaching value sharing

The doctor as the focus

- Communicating important shared values to patients or potential patients

- Stigmatisation and attitude change

- Personal identity

- Characteristics of good and bad doctors and good and bad people

- Rewards for work

heterosexual. This analytical approach has been used because each doctor explicitly identified his sexual preference to the interviewer without being asked: by chance three of the six participants identified as heterosexual, Drs B, C, \& D, and three as homosexual, Drs A, E, \& F. (In order to protect the identity of the participants, one of the first six letters of the alphabet has been randomly assigned to each doctor as a pseudonym.) It was also clear that the doctors made their sexual preference clear to their patients. The decision on the part of the doctors to make their sexual preference clear implies an investment of significance in the perceived relation between the care of HIV positive patients and the sexual identification of the carer. The respondents made it clear that sexual identification mattered.

When the data were analysed by comparing the views of homosexual and heterosexual doctors, the doctors' responses could be seen to vary with their own sexual orientation. The heterosexual doctors' responses were more similar to each other than to the responses of the homosexual doctors on various questions, and in particular on value sharing. The same was true for the homosexual doctors. 


\section{Value sharing}

In analysing the discussion about "values" and "value sharing," it became clear that each of the doctors had made the assumption that the values of HIV positive patients were principally the values of homosexuality, and that providing care to HIV positive patients effectively meant providing care to gay men. This perspective can easily be understood when viewed in light of the fact that an estimated $76-80 \%$ of HIV positive patients in South Australia contracted the infection through male to male sexual contact. As a consequence, for all of the respondents, the discussion of "values" and "value sharing" was mainly considered in the context of homosexual culture.

In response to questions about whether or not they believed they shared values with their HIV positive patients, the gay-identified doctors each agreed that they did, at least to some extent:

Certainly I think my own set of values around these issues are within the spectrum. They wouldn't be at any particular end of the spectrum but the Venn diagrams would cross over in many areas. (Dr A)

At a kind of basic level, like at a fundamental level where you look at a person and say, "am I generally the same or am I different?" then yes I'm the same, or we do have similar values in common. (Dr E)

The heterosexual doctors, by contrast, seemed to have some difficulty understanding the meaning of the word "value":

It depends what you call 'values'... (Dr B)

What do you mean 'value?' What are you talking about? ... I don't like the word 'value.' (Dr D)

Despite their uncertainty about the nature of "values", the heterosexual doctors were able to say that they did not share the values of their HIV positive patients.

Question: do you find when you're dealing with them that you see elements of your own value system in the patients? ... Do you see bits of it?

Response: not really, no. (Dr B)

The homosexual doctors did not share this uncertainty surrounding "values" and "value sharing". Indeed, the homosexual doctors saw "homosexuality" as a cultural label and then proceeded to deconstruct the label to try to explore the values underpinning it. The underlying values they identified as being important included the following: being more "liberal," more "radical," more "pursuant of independence", and more concerned with "sexual freedom."

It is understandable that the doctors who identified with the homosexual label, and by implication with the culture it describes, should be more aware of the meanings that label carries. Their own participation in that culture would certainly inform their understanding of it, whereas the heterosexual doctors remain external to it and appreciate only the obviously visible or readily identifiable components-for example, behaviours.

It is important to note here that, in identifying as homosexual, these doctors may have then projected the values they held as the values of homosexuality. There is no evidence from these interviews to suggest that the values they perceived are in fact the values of homosexuality, or even of the patients in whom they perceived them. The temperance with which they applied the values they had described to themselves though, suggests that this was probably not the case. Instead, it seems more probable that their perception of shared values was based on a sense of identification with their patients. The assertion by all three homosexual doctors that there is a vast "range of values" to be found within homosexual culture further substantiates this view.

\section{The importance of shared values}

With respect to the role that shared values play in the care of patients, all six participants agreed that sharing patients' values was not essential in the provision of optimal care to HIV positive patients. The position on which there was disagreement, however, was the relative importance of value sharing.

The homosexual doctors believed that value sharing, although not necessary, was an important aspect of the relationship they developed with HIV positive patients:

It certainly makes my life easier because I don't have to be so vigilant about being non-judgmental ... Behaviours, terminologies, cultural institutions ... [patients] say it would be uncomfortable to have to explain those things to someone who isn't culturally aware. ( $\operatorname{Dr} A)$

But yes, I would have to say it is important without being essential. It makes life easier for both the patient and the doctor ... As a gay doctor I understand gay issues, gay life, the difficulty of being gay ... so yes, I think it helps a lot. (Dr E)

People want to feel they connect with you, that you share in their suffering because ultimately that's the most valuable thing you can do for someone-share with them. (Dr F)

Two of the heterosexual doctors believed that sharing values with patients would be a useful clinical facility:

There has to be a greater understanding and rapport to start with. (Dr B)

I think a gay doctor probably has a closer to perfect relationship ... I think the lack of shared experience makes [patients] think that they can't say something. ( $\mathrm{Dr} C$ )

Doctor B and C, however, did not believe that not sharing values harmed the clinical relationship. They had a clear sense that, with a commitment to understanding and acceptance, value sharing as a source of identification was not necessary. They also suggested that drawing parallels in an attempt to identify with the patient was an effective way to build a good relationship with their patients:

It doesn't actually mean that there is any better relationship between them, though ... I'm accepting of everything really. We've all got biases ... but they have the feeling that I'm open to all those values ... I think they feel comfortable talking to me about those things. (Dr B) I'm not gay, but I think you can always turn things around and say, "what's important to [me] as far as [my] sexuality is concerned? What's important about [my] lifestyle?" । think you have to be able to use your own experiences to relate in some way to other people's lifestyles ... you can always say, "I know what I value," and use that to understand. (Dr C)

Dr D took a different perspective to each of the other five respondents. He believed that it was accepted among doctors that their values and attitudes should never enter the clinical encounter and as such, any discussion of value sharing is 
meaningless: "He sends her off to Dr Bloggs, gets [an abortion] done and comes back and the doctor then doesn't like that patient or can't empathise because he has made a value judgement about her. That's wrong. Doctors don't do that ... because to me it doesn't matter what the doctor's views are. That is irrelevant. I think the doctor's views, feelings don't come into patient care ... If a doctor has difficulty giving something to the patient, which is what you are talking about, it's better to be up front and say, "can't do it." But the patient is made up of lots of bits and it may be just that bit which he can't supply, so patient care still continues." (Dr D)

Dr D also advanced an equally emphatic defence of the difficulties facing doctors as human beings: "...there are doctors who have "blind spots." But you can't blame them. You can't be punitive. You can't point the finger at them. You can't say, "He's a pig because he has a blind spot about abortion. He's a bastard..." We need to be empathic and humane towards our patients but also towards each other. You need to understand what happens to us. I have very strong views about that...". (Dr D)

\section{The features patients look for in their doctors}

All of the respondents identified a number of similar features they believed HIV positive patients desired in their doctors. "Non-judgmental acceptance" and "understanding" were the common themes.

You need openness and honesty ... Probably I should have said this first but just, you know, listening to the patient is important ... I mean actually hearing what they're saying and sometimes hearing what they're not saying. (Dr E) Well, the first thing they look for is someone who is going to be sympathetic to the disease and to the homosexuality. Always first. Secondly, someone who is knowledgeable because it is a very specialised area. And thirdly, somebody who is a caring, understanding, listening type. (Dr D)

You need flexibility, a non-judgemental approach ... (Dr B)

... the ability to sit with them, share with them in their suffering, accept what they are saying. That is extremely important. (Dr F)

... wanting to be listened to ... they want not to be judged or disapproved of. (Dr A)

Confidentiality was also reported to be an important concern:
A lot of them want to know that you're going to keep confidentiality. (Dr B)
Confidentiality is a big one too. That's important to patients. (Dr E)

Expertise was rated as being particularly important by some of the doctors, especially those who identified themselves as heterosexual.

\begin{abstract}
I think they are looking for people who will listen to them so that they can explain their situation and who can give them the answers they want. (Dr C)

Obviously they want to have access to someone who is well versed with HIV, particularly therapy. (Dr B)
\end{abstract}

The doctors identified as homosexual were less likely to offer this view; they tended to view understanding and acceptance as paramount, with other features like expertise assuming secondary importance.

\section{Role of teaching}

The importance of building trust by accepting and understanding patients was emphasised by the participants, but these qualities were viewed as personality traits that a person either possessed or did not. They could not be taught in medical school, nor did everyone have the capacity to learn to be accepting or understanding of diversity.

I don't think you can teach it ... you can't change their head. You can teach someone as much as you like but it doesn't have to sink in. (Dr D)

It depends on the skills of the individual to be able to take that information and use it and the preparedness of the individual to be able to use it. ( $\operatorname{DrC}$ )

You can say you can learn [understanding] but you can't really ... I think it needs life experience more than anything. (Dr B)

You can learn to improve or at least provide a more overt demonstration of acceptance but learning is based on interest. Some people are interested and curious about difference; they're the ones who will learn. (Dr F)

\section{DISCUSSION}

All six doctors in this study assumed that the value systems of HIV positive patients could be equated with the value systems of homosexual people. The reason for why this may have happened has already been explained. It is questionable, however, whether this link is valid. Even in view of the fact that the most of the HIV positive patients these doctors see are GHAM, there may well be important value differences between those GHAM who contract HIV and those who do not. It seems overly simplistic to equate the value systems of these two groups of patients. This is especially important in the context of HIV where the attribution of negative values to HIV positive patients is, in part, responsible for their remaining a stigmatised and marginalised group. ${ }^{20}$ It is important that doctors realise the potential for attributing inappropriate values to patients, and the danger this may present if they are negative values.

This assumption notwithstanding, the doctors clearly divided themselves into two groups based on their sexual identification, and conferred upon this division a high degree of importance. On some issues the two groups agreed, whereas on others there was well defined disagreement. Most important in view of the aim of this study was the agreement among the doctors that value sharing is important, but not essential, to the provision of optimal health care to HIV positive patients. Disagreement arose between the two groups with respect to the relative importance of value sharing.

For the gay doctors, value sharing was a means of identification with their patients that allowed them to create the trusting and safe relationship they described as desirable. In perceiving a sense of shared values, these doctors believed that their gay patients' fear of rejection, which is said to be common among HIV positive patients, ${ }^{21}$ was immediately diminished by the knowledge that their doctor was also gay.

The heterosexual doctors believed that value sharing was a useful tool in developing the clinical relationship. However, they did not believe that the absence of shared values necessarily precluded the development of an appropriate and therapeutic relationship. More than the homosexual doctors, these doctors believed skill and expertise to be important for the creation of a safe and trusting environment. Building parallels between one's own life and that of the patient was also seen as a useful tool, but the ideas "being understanding" and "just accepting" the patient were the most 
common themes used to describe the development of trust. Although patients were not interviewed in this study, the finding that non-judgmental understanding and acceptance were important is consistent with the literature concerning HIV positive patients' desired features of medical care. ${ }^{22} 23$

None of the doctors in this study used the word "trust" in their discussion of the features of medical relationships, but this notion was implicit in the view that understanding and acceptance were the salient qualities of doctors. As trusting is considered a risk taking behaviour, ${ }^{24}$ it can be assumed that allowing the patient to feel understood, and accepting the information he or she presents without judgement, are attitudes employed to make the patient believe in the security of the clinical environment, and hence, trust in the doctorpatient relationship.

\section{CONCLUSION}

In this study, sexual orientation was deemed to be the shared value for relationships between these doctors and their patients. This finding may imply at least two things. Firstly, sexual orientation may be a catchall for a range of values. The doctors in our study alluded to this, when they noted that homosexuality was a cultural label that stood for being "liberal", "radical", "pursuant of independence", and concerned with "sexual freedom". Secondly, it may be the case that sexual orientation is such a powerful value that it overshadows other values that may also be shared, but less commonly acknowledged.

It is, of course, not possible to draw statistically significant conclusions from this empirical study, but the results presented here do represent an attempt to marry the theoretical discussion around value sharing with its place in practice. On the basis of this study, value sharing (as in Veatch's model) can be seen as a theoretically and practically important concept. However, at least for the participants in this study, it could not be regarded as essential to the clinical relationship.

Clearly, there are limits to the generalisability of a study such as this. HIV transmission in South Australia is predominantly through male to male sexual contact, and this provides a cultural setting in which linking the values of HIV positive men with gay culture makes sense. In addition, HIV/AIDS is an illness where the mode of transmission is clearly linked to sexuality and sexual practices. A comparable study where patients were heterosexual patients of African origin, or injecting drug users, or where patients were presenting for care of their cardiac disease, would likely lead to rather different findings.

The study also suggests other important directions for future research. The findings presented in this paper are, in some ways, rather one sided, as they reflect only the views that these doctors held about their patients. No evidence exists concerning the long term health outcomes for HIV positive patients on the basis of their satisfaction with the clinical relationship. The question that needs to be asked is whether the patients believe shared values to be important and how this influences long term health outcomes. Comparative studies are needed to address this question.

Another important consideration is in the context of medical education. It has been reported that student attitudes toward HIV positive patients become more negative as they progress through medical school. ${ }^{25}$ Clearly, there is a need for medical educators to nurture appreciation for diversity in their students and provide sources of inspiration for the maintenance of value systems that support the acceptance of difference. An investigation of potential techniques for encouraging medical students to a "willingness to learn" about understanding and acceptance of diversity would be of immense benefit. In this way, students enrolled in medical courses could bring with them a range of personal strengths but, at the same time, develop new skills to satisfy the community's desire for doctors who are able to provide a safe and trustworthy clinical environment to all the patients who present to them.

\section{Authors' affiliations}

A Lawlor, Department of Public Health, University of Adelaide, SA 5005, Australia

A Braunack-Mayer, Department of Public Health, University of Adelaide, SA 5005, Australia

\section{REFERENCES}

1 Hart SL. Axiology-Theory of values. Philosophy and Phenomenological Research 1971;22:29

2 Hermans HJM, Oles PK. The personal meaning of values in a rapidly changing society. Journal of Social Psychology 1994;134:569-79.

3 Gortzen R. Duty and inclination: the phenomenological value ethics of Hans Reiner. Journal of Value Inquiry 1991;25:119-45.

4 Von-Mering O. A grammar of human values. Pittsburgh: Pittsburgh University Press, 1991.

5 Misztal M. Reflections on the study of value systems. Polish Sociological Bulletin 1979; 1:45-51.

6 Mullin BA, Hogg MA. Motivations for group membership: The role of subjective importance and uncertainty reduction. Basic and Applied Social Psychology 1999;21:91-102.

7 Marques JM, et al. The role of categorization and in-group norms in judgements of groups and their members. Journal of Personality and Social Psychology 1998;75:976-88.

8 Alexander J. Beyond identity: queer values and the community. Journal of Gay, Lesbian, and Bisexual Identity 1999:4:293-314.

9 Veatch RM. The patient-physician relation: the patient as partner, part 2. Bloomington and Indianapolis: Indiana University Press, 1991;51.

10 See reference 9:4.

11 See reference 9:4.

12 See reference 9:51

13 See reference 9:47-51

14 Veatch RM. Abandoning informed consent. Hastings Cent Rep 1995;25:5-12.

15 Jacobs JA, Tedford-Jr JH. Factors affecting the self-esteem of the homosexual individual. J Homosex 1980;5:373-82.

16 Fassinger RE, Miller BA. Validation of an inclusive model of sexual minority identity formation on a sample of gay men. J Homosex 1996;32:53-78.

17 Gerbert B, et al. Primary care physicians and aids: attitudinal and structural barriers to care. JAMA 1991;266:2837-42.

18 Forrester D, Murphy P. Nurses' attitudes toward patients with aids and aidsrelated risk factors. J Adv Nurs 1992;17:1260-6.

19 Richards T, Richards L. The NUDIST qualitative data analysis system. Qualitative Sociology 1991;14:307-24.

20 Kurtz ME, et al. Teaching medical students the effects of values and stereotyping on the doctor/patient relationship. Social Science and Medicine 1985;9:1043-7.

21 King MB. AIDS and the general practitioner: views of patients with HIV infection and AIDS. BMJ 1988;297:182-4.

22 Kochen MM, et al. How do patients with HIV perceive their general practitioners? BMJ 1991;303:1365-8.

23 Mansfield SJ, Singh S. The general practitioner and human immunodeficiency virus infection: An insight into patients' attitudes. J R Coll Gen Pract 1989;39:104-5.

24 Govier T. An epistemology of trust. International Journal of Moral and Social Studies 1993;8:155-74.

25 Weyant RJ, et al. Changes in medical students' attitudes toward HIV-infected patients as the students progress through medical school. Acad Med 1993;68:377-9. 